University of Nebraska - Lincoln

DigitalCommons@University of Nebraska - Lincoln

$9-22-2006$

\title{
Elliptic and Circular Dichroism Effects in Two-Photon Double Ionization of Atoms
}

Andrei Y. Istomin

University of Nebraska - Lincoln

Evgeny Pronin

University of Nebraska - Lincoln, pronin@phys.vsu.ru

N. L. Manakov

Voronezh State University, manakov@phys.vsu.ru

S.I. Marmo

University of Nebraska - Lincoln

Anthony F. Starace

University of Nebraska-Lincoln, astarace1@unl.edu

Follow this and additional works at: https://digitalcommons.unl.edu/physicsstarace

Part of the Physics Commons

Istomin, Andrei Y.; Pronin, Evgeny; Manakov, N. L.; Marmo, S.I.; and Starace, Anthony F., "Elliptic and Circular Dichroism Effects in Two-Photon Double Ionization of Atoms" (2006). Anthony F. Starace Publications. 97.

https://digitalcommons.unl.edu/physicsstarace/97

This Article is brought to you for free and open access by the Research Papers in Physics and Astronomy at DigitalCommons@University of Nebraska - Lincoln. It has been accepted for inclusion in Anthony F. Starace Publications by an authorized administrator of DigitalCommons@University of Nebraska - Lincoln. 


\title{
Elliptic and Circular Dichroism Effects in Two-Photon Double Ionization of Atoms
}

\author{
Andrei Y. Istomin, ${ }^{1, *}$ E. A. Pronin,,${ }^{1,2}$ N. L. Manakov, ${ }^{2}$ S. I. Marmo, ${ }^{2}$ and Anthony F. Starace ${ }^{1}$ \\ ${ }^{1}$ Department of Physics and Astronomy, The University of Nebraska, Lincoln, Nebraska 68588-0111, USA \\ ${ }^{2}$ Department of Physics, Voronezh State University, Voronezh 394006, Russia
}

(Received 3 May 2006; published 22 September 2006)

\begin{abstract}
An $a b$ initio parametrization of the two-photon double ionization amplitude from an $s^{2}$ subshell of an atom in a ${ }^{1} S$ state is presented and used to predict two light polarization effects on photoelectron angular distributions that do not exist in single-photon double ionization: (i) elliptic dichroism and (ii) circular dichroism at equal energy sharing. Estimates for He show large magnitudes for these effects, which provide a means for polarization control of double ionization by vacuum ultraviolet light.
\end{abstract}

The physics of processes involving simultaneous ejection of several electrons from atoms and molecules has attracted interest since the 1960 s owing to the insight into electron correlations that such studies provide. Singlephoton double ionization (SPDI) of He (which can be measured in electron-electron or electron-recoil ion coincidence experiments) has been a research focus over the past decade. At present there exists a wealth of knowledge concerning electron correlations for this fundamental three-body problem [1]. Recent advances in generating vuv light of higher intensity (than provided by synchrotrons) make possible studies of multielectron ejection due to multiphoton interactions of atomic electrons with vuv radiation. Two-photon double ionization (TPDI) of He has recently attracted much attention, as its study provides new insights into two-electron ejection dynamics (see, e.g., Refs. [2-5]). In particular, in contrast to SPDI, in which the $P$-wave channel of a two-electron continuum is populated, the TPDI process allows one to probe the $S$ - and $D$-wave channels. The study of TPDI also provides a "bridge" between SPDI and multiphoton nonsequential double ionization (NSDI) in optical laser fields [6]. Experimentally, TPDI of $\mathrm{He}$ was investigated using the 27th harmonic of a Ti:sapphire laser with a photon energy of $41.8 \mathrm{eV}$ and a peak intensity of $10^{14} \mathrm{~W} / \mathrm{cm}^{2}$ [7]. So far all studies of TPDI have been limited to the case of linearly polarized light.

In this Letter, we study how the shape of the photoelectron angular distribution in TPDI [i.e., of the triplydifferential cross section (TDCS)] depends upon the handedness of (in general elliptically polarized) vacuum ultraviolet (vuv) radiation. In SPDI, this dependence was established in 1992 [8], and is termed the circular dichroism (CD) effect; it is maximal for circularly polarized light and vanishes when two electrons are ejected with equal energies. In TPDI, we show that in addition to the CD effect, there exists also a qualitatively different, elliptic dichroism (ED) effect: in particular geometries that we specify, the shape of the TDCS depends on the helicity of the vuv light only for elliptic polarization (i.e., the effect disappears for pure circular polarization). We also show that in TPDI by circularly polarized light (i.e., when the ED effect vanishes), the $\mathrm{CD}$ effect persists even when both electrons are ejected with equal energies. In our numerical estimates of the TDCS for nonsequential TPDI of He, we consider photons of energy $45 \mathrm{eV}$ (the 29th harmonic of a Ti:sapphire laser) and take into account their interaction with He perturbatively, which is adequate up to intensities of the order of $10^{14} \mathrm{~W} / \mathrm{cm}^{2}$ [4].

The TDCS for TPDI of an atom by radiation having an electric field vector $\mathbf{F}(\mathbf{r}, t)=F \operatorname{Re}\{\mathbf{e} \exp [i(\mathbf{k} \cdot \mathbf{r}-\omega t)]\}$ $\left(\mathbf{e} \cdot \mathbf{e}^{*}=1\right)$ and a photon flux density $|\mathbf{j}|=c F^{2} /(8 \pi \hbar \omega)$ (defined as $d \sigma=d W /|\mathbf{j}|^{2}$, where $d W$ is the transition rate) is given in atomic units by

$$
d^{3} \sigma /\left(d \Omega_{1} d \Omega_{2} d E_{2}\right) \equiv \sigma=\mathcal{A}|A|^{2},
$$

where $\mathcal{A}=8 \pi^{3} p_{1} p_{2} /\left(c^{2} \omega^{2}\right), p_{1}$ and $p_{2}$ are the photoelectron momenta, and $E_{2}=p_{2}^{2} / 2$. The amplitude $A$ for a two-photon transition from an initial $s^{2}\left({ }^{1} S_{0}\right)$ state $\Phi_{i}$ to the two-electron continuum state $\Psi_{\mathbf{p}_{1} \mathbf{p}_{2}}^{(-)}$involves the twoelectron Green's function (TEGF) $G\left(E_{i}+\omega\right)$,

$$
A=\left\langle\Psi_{\mathbf{p}_{1} \mathbf{p}_{2}}^{(-)}\left|(\mathbf{e} \cdot \mathbf{D}) G\left(E_{i}+\omega\right)(\mathbf{e} \cdot \mathbf{D})\right| \Phi_{i}\right\rangle,
$$

where $(\mathbf{e} \cdot \mathbf{D})=\mathbf{e} \cdot\left(\nabla_{\mathbf{r}_{1}}+\nabla_{\mathbf{r}_{2}}\right)$ is the electric dipole operator (in velocity form) of the electron-photon interaction.

Using the standard expansion of the final state wave function and the TEGF in Eq. (2) in bipolar harmonics [9], the evaluation of angular integrals in Eq. (2) by means of the Wigner-Eckart theorem yields

$$
A=\sum_{L=0,2} \sum_{\substack{l_{1} l_{2} \\ s_{1} s_{2}}} \frac{d_{l_{1} l_{2}(L)}^{s_{1} s_{2}}}{\sqrt{3(2 L+1)}}\left[\{\mathbf{e} \otimes \mathbf{e}\}_{L} \cdot Y_{L}^{l_{1} l_{2}}\left(\hat{\mathbf{p}}_{1}, \hat{\mathbf{p}}_{2}\right)\right],
$$

where $d_{l_{1} l_{2}(L)}^{s_{1} s_{2}} \equiv\left\langle p_{1} p_{2}\left(l_{1} l_{2}\right) L\left\|\mathbf{D} g_{1}^{s_{1} s_{2}}\left(E_{i}+\omega\right) \mathbf{D}\right\| \Phi_{i}\right\rangle$ is the second-order two-electron reduced matrix element in which $g_{1}^{s_{1} s_{2}}(\mathcal{E})$ describes "intermediate" states of the electron pair of ${ }^{1} P^{o}$ symmetry with orbital angular momenta $s_{1}$ and $s_{2}\left(s_{1}+s_{2}=\right.$ odd and $l_{1}+l_{2}=$ even due to parity conservation). After rewriting the bipolar harmonic $Y_{L}^{l_{1} l_{2}}\left(\hat{\mathbf{p}}_{1}, \hat{\mathbf{p}}_{2}\right)$ in Eq. (3) in terms of irreducible tensors composed of vectors $\hat{\mathbf{p}}_{1}$ and $\hat{\mathbf{p}}_{2}$ [10] and evaluating the 
scalar product of these tensors with photon polarization tensors $\{\mathbf{e} \otimes \mathbf{e}\}_{L}$ [9], we obtain the following invariant form for the TPDI amplitude:

$$
A=f_{0}(\mathbf{e} \cdot \mathbf{e})+f_{1}\left(\mathbf{e} \cdot \hat{\mathbf{p}}_{1}\right)^{2}+f_{2}\left(\mathbf{e} \cdot \hat{\mathbf{p}}_{2}\right)^{2}+f_{s}\left(\mathbf{e} \cdot \hat{\mathbf{p}}_{1}\right)\left(\mathbf{e} \cdot \hat{\mathbf{p}}_{2}\right),
$$

where $f_{0}\left(p_{1}, p_{2}\right), f_{1}\left(p_{1}, p_{2}\right), f_{2}\left(p_{1}, p_{2}\right) \equiv f_{1}\left(p_{2}, p_{1}\right)$, and $f_{s}\left(p_{1}, p_{2}\right)$ are four polarization-invariant amplitudes that depend only upon the photoelectron energies and $x \equiv$ $\cos \theta=\left(\hat{\mathbf{p}}_{1} \cdot \hat{\mathbf{p}}_{2}\right)$. They have the following general expressions in terms of Legendre polynomials, $P_{l}(x)$, and their derivatives, $P_{l}^{(k)}(x)=(d / d x)^{k} P_{l}(x)$ :

$$
\begin{gathered}
f_{1}=\sum_{l=2}^{\infty}\left[\sqrt{\frac{2(2 l+1)}{(2 l-1) l(l+1)(2 l+3)}} M_{l l}^{2}\right. \\
-\frac{1}{\sqrt{3(l+1)(l+2)(2 l+3)}} M_{l l+2}^{2} \\
\left.\quad-\frac{1}{\sqrt{3(l-1) l(2 l-1)}} M_{l l-2}^{2}\right] P_{l}^{(2)}(x), \\
f_{s}=\sum_{l=1}^{\infty} \frac{2}{\sqrt{l(l+1)(2 l+1)}} \\
\times\left\{\frac{2 l+1}{\sqrt{2(2 l-1)(2 l+3)}} M_{l l}^{2}\left[2 x P_{l}^{(2)}(x)-P_{l}^{(1)}(x)\right]\right. \\
\left.+\frac{1}{\sqrt{3}}\left(M_{l-1 l+1}^{2}+M_{l+1 l-1}^{2}\right) P_{l}^{(2)}(x)\right\}, \\
f_{0}=-\frac{1}{3}\left[\sum_{l=0}^{\infty} \sqrt{2 l+1} M_{l l}^{0} P_{l}(x)+f_{1}+f_{2}+x f_{s}\right] .
\end{gathered}
$$

In Eqs. (5)-(7), $M_{l_{1} l_{2}}^{L} \equiv(4 \pi)^{-1} \sum_{s_{1}=0}^{\infty} \sum_{s_{2}=s_{1} \pm 1} d_{l_{1} l_{2}(L)}^{s_{1} s_{2}}$ is a dynamical factor relevant to the $L$-wave continuum channel. It depends only upon photoelectron energies and the dynamical model used to describe electron correlations. Note that the $S$-wave continuum channel factors, $M_{l_{1} l_{2}}^{0}$, enter only the amplitude $f_{0}$, which does not contribute in the case of circular polarization [since $(\mathbf{e} \cdot \mathbf{e})=0$ for this case]. The amplitudes $f_{s}$ and $f_{0}$ are symmetric in $p_{1}, p_{2}$ : $f_{0}\left(p_{1}, p_{2}\right)=f_{0}\left(p_{2}, p_{1}\right), f_{s}\left(p_{1}, p_{2}\right)=f_{s}\left(p_{2}, p_{1}\right)$. By introducing $g_{s, a}=f_{1} \pm f_{2}$, we obtain an alternative set of four symmetrized amplitudes, where the antysimmetric amplitude, $g_{a}$, vanishes at equal energy sharing, $p_{1}=p_{2}$. The parametrization of $A$ in Eq. (4) is similar to that for absorption of two photons in laser-assisted electron-atom scattering [11] (because the same set of vectors occurs in both problems). Note that an alternative parametrization of the TPDI amplitude [5] involves five parameters, indicating an implicit redundancy.

To analyze the TDCS, we parametrize the photon polarization vector as $\mathbf{e}=(\hat{\boldsymbol{\epsilon}}+i \eta \hat{\zeta}) / \sqrt{1+\eta^{2}}(-1 \leq \eta \leq 1)$, where $\hat{\boldsymbol{\zeta}}=[\hat{\mathbf{k}} \times \hat{\boldsymbol{\epsilon}}]$ and $\hat{\boldsymbol{\epsilon}}$ and $\hat{\mathbf{k}}$ indicate the directions of the major axis of the polarization ellipse and the photon wave vector, $\mathbf{k}$. The ellipticity $\eta$ is related to the degrees of linear and circular polarization, $l$ and $\xi: l=\left(1-\eta^{2}\right) /$ $\left(1+\eta^{2}\right)=(\mathbf{e} \cdot \mathbf{e}), \quad \xi=2 \eta /\left(1+\eta^{2}\right)=i\left(\hat{\mathbf{k}} \cdot\left[\mathbf{e}^{*} \times \mathbf{e}\right]\right) ;$ $l^{2}+\xi^{2}=1$. With these definitions and Eq. (4), the TDCS in Eq. (1) may be expressed as (cf. [11]):

$$
\sigma=\sigma_{0}+\xi \sigma_{\mathrm{CD}}+\xi l \sigma_{\mathrm{ED}},
$$

where $\sigma_{0}$ is invariant under the transformation $\mathbf{e} \rightarrow \mathbf{e}^{*}$ (i.e., under a change in sign of $\xi$ ), while the two other terms are dichroic (i.e., they change sign when $\xi \rightarrow-\xi$ ). The term proportional to $\xi$ is maximal at $\xi= \pm 1$ and describes the $\mathrm{CD}$ effect, while the term proportional to $\xi l$ vanishes for purely circular polarization $(\xi= \pm 1, l=0)$ and describes the ED effect; it is maximal for $l=|\xi|=$ $1 / \sqrt{2}$. The general expressions for $\sigma_{0}, \sigma_{\mathrm{CD}}$, and $\sigma_{\mathrm{ED}}$ in Eq. (8) for unequal energy sharing will be published elsewhere; $\sigma_{\mathrm{CD}}$ involves only the amplitudes $f_{s}, f_{1}$, and $f_{2}$, while $\sigma_{\mathrm{ED}}$ also involves $f_{0}$. Here we present explicit expressions for $\sigma_{\mathrm{CD}}$ and $\sigma_{\mathrm{ED}}$ for the case of equal energy sharing $\left[p_{1}=p_{2}, \mathbf{p}_{ \pm}=\left(\hat{\mathbf{p}}_{1} \pm \hat{\mathbf{p}}_{2}\right) / 2,\left(\mathbf{p}_{+} \cdot \mathbf{p}_{-}\right)=0\right]$ :

$$
\sigma_{\mathrm{CD}}^{(\mathrm{eq})}=2 \mathcal{A} \operatorname{Im}\left(f_{s}^{*} g_{s}\right)\left(\hat{\mathbf{k}} \cdot\left[\mathbf{p}_{+} \times \mathbf{p}_{-}\right]\right)\left(\hat{\mathbf{k}} \cdot \mathbf{p}_{+}\right)\left(\hat{\mathbf{k}} \cdot \mathbf{p}_{-}\right),
$$

$$
\begin{aligned}
\sigma_{\mathrm{ED}}^{(\mathrm{eq})}= & 4 \mathcal{A} \operatorname{Im}\left(g_{s}^{*} f_{s}\right)\left(\hat{\mathbf{k}} \cdot\left[\mathbf{p}_{+} \times \mathbf{p}_{-}\right]\right)\left(\hat{\boldsymbol{\epsilon}} \cdot \mathbf{p}_{+}\right)\left(\hat{\boldsymbol{\epsilon}} \cdot \mathbf{p}_{-}\right) \\
& +2 \mathcal{A} \operatorname{Im}\left[\left(f_{s}+g_{s}\right)^{*} f_{0}\right] \\
& \times\left[\left(\hat{\boldsymbol{\epsilon}} \cdot \mathbf{p}_{+}\right)\left(\hat{\boldsymbol{\zeta}} \cdot \mathbf{p}_{+}\right)+\left(\hat{\boldsymbol{\epsilon}} \cdot \mathbf{p}_{-}\right)\left(\hat{\boldsymbol{\zeta}} \cdot \mathbf{p}_{-}\right)\right]-\sigma_{\mathrm{CD}}^{(\mathrm{eq})}
\end{aligned}
$$

One sees that dichroic effects in TPDI originate from interference of real and imaginary parts of polarizationinvariant components of the generally non-Hermitian TPDI amplitude, in agreement with arguments in Ref. [12] on the origin of dichroic effects in photoprocesses involving unpolarized atoms.

The parametrizations (4)-(10) constitute the main results of this Letter. In general, the dichroic terms (9) and (10) are not small compared to $\sigma_{0}$ in (8). Evaluating these terms is theoretically challenging. Existing calculations on TPDI treat only linearly polarized light, for which numerical solution of the Schrödinger equation represents a spatial five-dimensional problem; the various total cross section results for $\omega=45 \mathrm{eV}$ differ by more than a factor of 2 (cf. Fig. 1 of [5]). A direct numerical solution for elliptically polarized light represents a spatial sixdimensional problem. Accurate experimental measurements thus may provide a valuable benchmark for theory. In order to estimate the magnitudes of the $\mathrm{CD}$ and $\mathrm{ED}$ effects in TPDI of He for such experiments, we use lowestorder perturbation theory (LOPT) in the interelectron interaction, $1 / r_{12}$, to account for electron correlations, as done successfully to treat both NSDI by strong laser fields [6] and SPDI [13]. In SPDI, it was shown [13] that the knockout mechanism is dominant for excess energies of tens of $\mathrm{eV}$ and symmetric energy sharing. In the TPDI amplitude, there are two knockout terms, in which both 



FIG. 1. Schematic diagrams for the knockout contributions to the TPDI transition amplitude. The solid dots indicate summations over intermediate states. Diagrams with interchanged $\mathbf{p}_{1}$ and $\mathbf{p}_{2}$ must also be included.

photons are absorbed by the same electron; their diagrams are shown in Fig. 1. The diagram in Fig. 1(b) turns out to be negligible. Besides the knockout diagrams, the LOPT TPDI amplitude involves also diagrams corresponding to absorption of a single photon by each of the two electrons. Our estimates show that, for the photon energy considered in this Letter $(\sim 45 \mathrm{eV})$, these diagrams are suppressed compared to the knockout ones except in the narrow interval of angles $\theta$ near $\theta=0$. Moreover, to obtain correct results for this region of $\theta$, the inclusion of high-order effects in $1 / r_{12}$ is necessary owing to the divergence of these LOPT diagrams at equal energy sharing. Such calculations require the development of an appropriate regularization procedure and significant computational effort; thus we confine our estimates of dichroic effects in TPDI to those originating from knockout processes. These give the right order of magnitude for the TDCS in the case of linearly polarized photons, as shown in Fig. 2 where we make comparisons with the time-dependent close coupling (TDCC) [3] and convergent close coupling (CCC) [5] results. (Note that in Ref. [5] and here, the CCC results
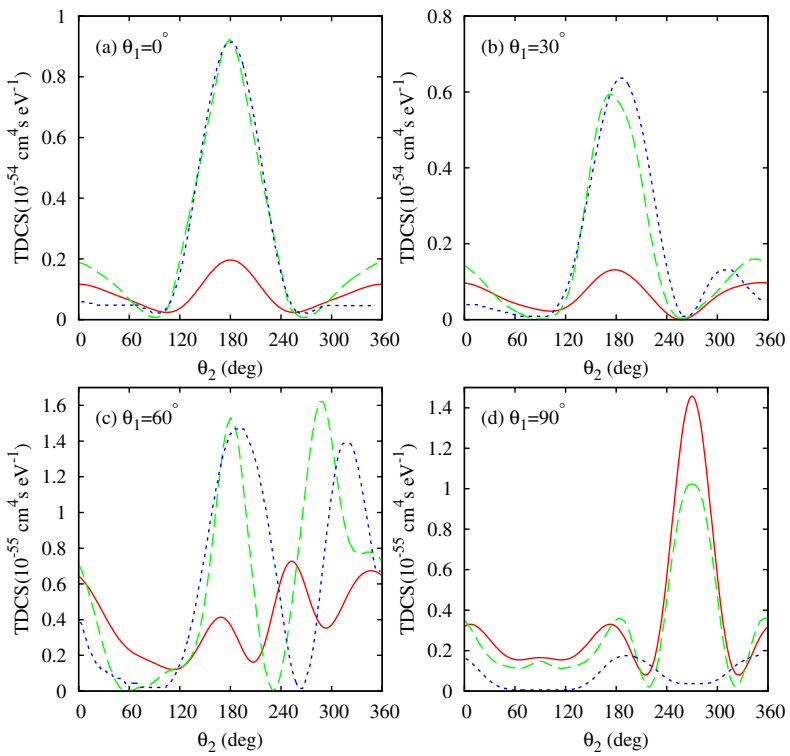

FIG. 2 (color online). TDCS for TPDI of He by linearly polarized photons of energy $\omega=45 \mathrm{eV}$. Full (red) curves, present LOPT knockout results; dotted (blue) curves, TDCC results [3]; dashed (green) curves, CCC results [5] scaled to the TDCC results as in [5].


FIG. 3 (color online). Geometries for measurement of the ED and equal energy sharing CD effects in TPDI. (a) Orthogonal geometry for measurement of ED at $p_{1}=p_{2}$. (b) Coplanar geometry for measurement of $\mathrm{ED}$, with one electron $\left(\mathbf{p}_{1}\right)$ detected in the $x y$ plane and the other $\left(\mathbf{p}_{2}\right)$ detected in the plane spanned by $\mathbf{k}$ and $\mathbf{p}_{1}$. (c) Geometry for measurement of CD at $p_{1}=p_{2}$ with fixed $\varphi_{2}$.

are scaled by a factor of 7). Our absolute LOPT estimates agree in order of magnitude with both the absolute TDCC and the scaled CCC results. (Our total TPDI cross section results for $40 \mathrm{eV} \leq \omega \leq 45 \mathrm{eV}$ - not shown-agree to within $\pm 50 \%$ with the absolute TDCC results.) Our predicted angular structure agrees qualitatively with both results for $\theta_{1}=0^{\circ}$ and $30^{\circ}$, while it agrees only with the scaled CCC results for $\theta_{1}=90^{\circ}$. For $\theta_{1}=60^{\circ}$ our LOPT
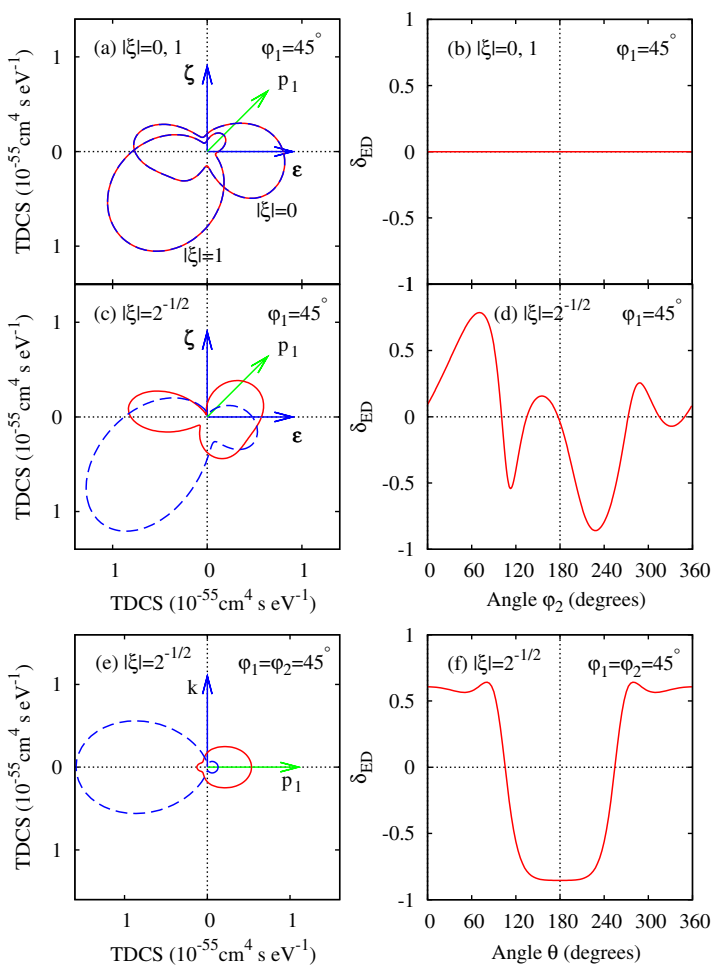

FIG. 4 (color online). TDCS, $\sigma$, and the ED parameter, $\delta_{\mathrm{ED}}$, for TPDI of He for equal energy sharing $\left(E_{1}=E_{2}=5.5 \mathrm{eV}\right)$. (a-d) Orthogonal geometry [Fig. 3(a)]. (e),(f) Coplanar geometry [Fig. 3(b)]. Left column: full (red) curves, $\sigma(|\xi|)$; dashed (blue) curves, $\sigma(-|\xi|)$. Right column: full (red) curves, $\delta_{\mathrm{ED}}$. 

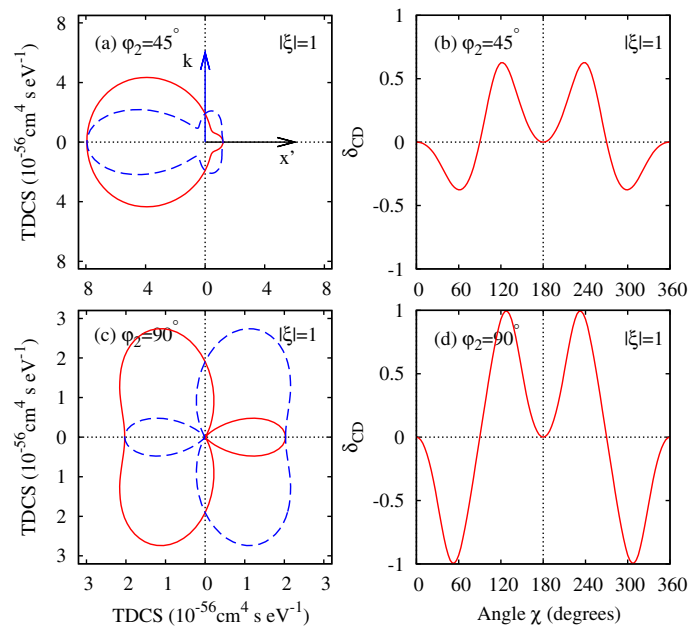

FIG. 5 (color online). Equal energy sharing CD effect for the geometry in Fig. 3(c). Parameters and curves are as in Fig. 4.

results predict a more compressed angular structure than do the others.

In Figs. 4 and 5 we present our LOPT estimates for the ED and CD effects, respectively, for three geometries in which only one of the effects is present [cf. Fig. 3]. The left column of Fig. 4 shows polar plots of TDCSs for lefthanded $(\xi>0)$ and right-handed $(\xi<0)$ photons, while the right column shows the relative ED parameter, $\delta_{\mathrm{ED}} \equiv$ $[\sigma(\xi)-\sigma(-\xi)] /[\sigma(\xi)+\sigma(-\xi)]$, as a function of the corresponding polar angles. In Figs. 4(a)-4(d) we use the orthogonal geometry, while in Figs. 4(e) and 4(f) we use the coplanar geometry. Figure 4(d) has two geometrical zeros determined by the condition that one of the vectors $\mathbf{p}_{+}$and $\mathbf{p}_{-}$is orthogonal to either $\hat{\boldsymbol{\epsilon}}$ or $\hat{\boldsymbol{\zeta}}$ (i.e., $\varphi_{2}=$ $180^{\circ}-\varphi_{1}$ or $\varphi_{2}=360^{\circ}-\varphi_{1}$, so that $\varphi_{2}=135^{\circ}$ or $315^{\circ}$ for $\varphi_{1}=45^{\circ}$ ); there are also four dynamical zeros. These zeros are caused by cancellations of terms involving $\operatorname{Im}\left(g_{s}^{*} f_{s}\right)$ and $\operatorname{Im}\left[\left(f_{s}+g_{s}\right)^{*} f_{0}\right]$ in Eq. (10): their positions within the same geometry depend on the excess energy. Two zeros (symmetric with respect to $\theta=180^{\circ}$ ) of $\delta_{\mathrm{ED}}$ in Fig. 4(f) also have a dynamical origin [since $\left(\hat{\mathbf{k}} \cdot\left[\mathbf{p}_{+} \times\right.\right.$ $\left.\left.\mathbf{p}_{-}\right]\right)=0$ for this geometry, they originate from a zero of the factor $\operatorname{Im}\left[\left(f_{s}+g_{s}\right)^{*} f_{0}\right]$, whose position depends on the excess energy].

The important difference between the CD effect in SPDI and TPDI is that CD in SPDI vanishes for equal energy sharing, while in TPDI it remains nonzero (except in orthogonal and coplanar geometries). In Fig. 5, we present TDCSs and CD parameters, $\delta_{\mathrm{CD}} \equiv[\sigma(+1)-$ $\sigma(-1)] /[\sigma(+1)+\sigma(-1)]$, for two cases having the geometry in Fig. 3(c): $\varphi_{2}=45^{\circ}$ and $\varphi_{2}=90^{\circ}$. All zeros of $\delta_{\mathrm{CD}}$ have geometrical origins; that means that the term $\operatorname{Im}\left(f_{s}^{*} g_{s}\right)$ in (9) does not change its sign in the interval $0^{\circ} \leq \chi<360^{\circ}$ [for $\varphi_{2}=90^{\circ}$, this fact is obvious since the mutual angle $\theta$ is fixed $\left(\theta=90^{\circ}\right)$ for this case].

To conclude, we have presented model-independent representations for the transition amplitude and the
TDCS for TPDI from the $s^{2}$ subshell of an atom in a ${ }^{1} S$ state. Based on these representations, we predict dichroic (ED and CD) effects that are nonzero for both symmetric and asymmetric excess energy sharing. Our analysis shows that the ED term in the TDCS involves a more feature-rich interference of polarization-invariant amplitudes than the CD term. Thus (in contrast to SPDI) measurements of TPDI with elliptically polarized light (with $0<|\xi|<1$ ) are more informative. Our LOPT knockout estimates for He show that both CD and ED effects should be observable in TDCS measurements.

This work was supported in part by the U.S. Department of Energy, Office of Science, Division of Chemical Sciences, Geosciences, and Biosciences, under Grant No. DE-FG03-96ER14646 and by RFBR Grant No. 0402-16350.

*Present address: Dept. Physics and Optical Science, Univ. of North Carolina, Charlotte, NC 28223, USA.

[1] J.S. Briggs and V. Schmidt, J. Phys. B 33, R1 (2000); L. Avaldi and A. Huetz, J. Phys. B 38, S861 (2005).

[2] M.S. Pindzola and F. Robicheaux, J. Phys. B 31, L823 (1998); M. A. Kornberg and P. Lambropoulos, J. Phys. B 32, L603 (1999); L. A. A. Nikolopoulos and P. Lambropoulos, J. Phys. B 34, 545 (2001); T. Mercouris, C. Haritos, and C. A. Nicolaides, J. Phys. B 34, 3789 (2001); S. Laulan and H. Bachau, Phys. Rev. A 68, 013409 (2003); L. Feng and H. W. van der Hart, J. Phys. B 36, L1 (2003); S. X. Hu, J. Colgan, and L. A. Collins, J. Phys. B 38, L35 (2005); I. F. Barna, J. Wang, and J. Burgdörfer, Phys. Rev. A 73, 023402 (2006).

[3] J. Colgan and M. S. Pindzola, Phys. Rev. Lett. 88, 173002 (2002).

[4] P. Lambropoulos, L.A.A. Nikolopoulos, and M.G. Makris, Phys. Rev. A 72, 013410 (2005); L. A. A. Nikolopoulos and P. Lambropoulos, J. Phys. B 39, 883 (2006).

[5] A. S. Kheifets and I. A. Ivanov, J. Phys. B 39, 1731 (2006).

[6] A. Becker and F. H. M. Faisal, J. Phys. B 38, R1 (2005).

[7] Y. Nabekawa, H. Hasegawa, E. J. Takahashi, and K. Midorikawa, Phys. Rev. Lett. 94, 043001 (2005); H. Hasegawa, E.J. Takahashi, Y. Nabekawa, K. L. Ishikawa, and K. Midorikawa, Phys. Rev. A 71, 023407 (2005).

[8] J. Berakdar and H. Klar, Phys. Rev. Lett. 69, 1175 (1992).

[9] D. A. Varshalovich, A. N. Moskalev, and V. K. Khersonskii, Quantum Theory of Angular Momentum (World Scientific, Singapore, 1988).

[10] N.L. Manakov, S. I. Marmo, and A. V. Meremianin, J. Phys. B 29, 2711 (1996).

[11] A. A. Krylovetsky, N. L. Manakov, S. I. Marmo, and A. F. Starace, Zh. Eksp. Teor. Fiz. 122, 1168 (2002) [JETP 95, 1006 (2002)].

[12] N. L. Manakov, M. V. Frolov, B. Borca, and A. F. Starace, J. Phys. B 36, R49 (2003).

[13] A. Y. Istomin, N. L. Manakov, and A. F. Starace, J. Phys. B 35, L543 (2002); Phys. Rev. A 69, 032713 (2004). 